\title{
In situ observations of a solar wind compression-induced hot plasma injection in Saturn's tail
}

\author{
E. J. Bunce, ${ }^{1}$ S. W. H. Cowley, ${ }^{1}$ D. M. Wright, ${ }^{1}$ A. J. Coates,${ }^{2}$ M. K. Dougherty, ${ }^{3}$ \\ N. Krupp, ${ }^{4}$ W. S. Kurth, ${ }^{5}$ and A. M. Rymer ${ }^{2}$ \\ Received 3 March 2005; revised 24 May 2005; accepted 13 June 2005; published 9 July 2005.
}

[1] During the Saturn orbit insertion (SOI) fly-through of the Cassini spacecraft, Saturn's magnetosphere underwent a significant corotating interaction region (CIR) related compression. Such compressions have recently been suggested to produce rapid bursts of tail reconnection, enhanced Saturn kilometric radiation (SKR), and consequent auroral dynamics. On the outbound pass the spacecraft became engulfed by hot plasma, associated with a reduction in field strength, and a change in orientation indicative of a dipolarisation. Concurrently, a substantial enhancement in SKR emissions took place, together with a disruption of the typical planetary modulation. We suggest this is the first in situ evidence of compression-related tail collapse via magnetic reconnection and hot plasma acceleration in Saturn's magnetotail. Citation: Bunce, E. J., S. W. H. Cowley, D. M. Wright, A. J. Coates, M. K. Dougherty, N. Krupp, W. S. Kurth, and A. M. Rymer (2005), In situ observations of a solar wind compression-induced hot plasma injection in Saturn's tail, Geophys. Res. Lett., 32, L20S04, doi:10.1029/2005GL022888.

\section{Introduction}

[2] The interplanetary medium upstream of Saturn is observed to be highly variable, consisting of few-day compression regions (characterized by high field strength and density) with embedded heliospheric current sheet (HCS) crossings, separated by several day rarefaction regions (characterized by low field strength and density). Jackman et al. [2004] have shown that this corotating interaction region (CIR) structure will strongly modulate the solar wind interaction at Saturn. Several authors have recently discussed the highly-dynamic nature of the aurora, and Saturn kilometric radiation (SKR), during the January 2004 Hubble Space Telescope (HST)-Cassini campaign with respect to CIR-related solar wind activity [Clarke et al., 2005; Crary et al., 2005; Grodent et al., 2005; Bunce et al., 2005]. Following a major compression region, the auroral oval was observed to expand significantly poleward, filling the whole of the dawn side polar cap region,

\footnotetext{
${ }^{1}$ Department of Physics and Astronomy, University of Leicester, Leicester, UK.

${ }^{2}$ Mullard Space Science Laboratory, University College London, Dorking, UK.

${ }^{3}$ Blackett Laboratory, Imperial College, London, UK.

${ }^{4}$ Max-Planck-Institut für Sonnensystemforschung, Katlenburg-Lindau, Germany.

${ }^{5}$ Department of Physics and Astronomy, University of Iowa, Iowa City, Iowa, USA.
}

Copyright 2005 by the American Geophysical Union. 0094-8276/05/2005GL022888 eventually settling into a bright 'spiral' extending from the nightside around dawn to the post-noon sector. The auroral oval brightening occurred at the same time as bursts of SKR emission and disruption of the usual diurnal pattern [Kurth et al., 2005]. Recently, Cowley et al. [2005] have proposed that these auroral and SKR effects are associated with CIR compression-induced tail reconnection, as is sometimes seen at Earth [e.g., Boudouridis et al., 2003]. This interval of strong tail reconnection would inevitably be accompanied by the injection of hot plasma into the nightside magnetosphere, and a significant reconfiguration of Saturn's magnetotail.

[3] Recently C. M. Jackman et al. (Interplanetary conditions and magnetospheric dynamics during the Cassini orbit insertion fly-through of Saturn's magnetosphere, submitted to Journal of Geophysical Research, 2005, hereinafter referred to as Jackman et al., submitted manuscript, 2005) have studied the interplanetary magnetic field and SKR emissions surrounding Saturn orbit insertion (SOI) fly-through, and suggest that a major compression of the magnetosphere took place during the pass. In this paper, we unify the observations made by the RPWS, MAG, MIMI-LEMMS, and CAPS-ELS instruments on the Cassini spacecraft during SOI, and show that outbound the spacecraft observed major field and plasma dynamics, suggestive of reconnection in the tail. Closer inspection of the magnetic field vectors during this time indicates a field relaxation, characteristic of major tail reconfigurations during substorms at Earth.

\section{Evidence for Magnetospheric Compression During SOI}

[4] Jackman et al. (submitted manuscript, 2005) have shown that a CIR-related compression took place whilst Cassini traversed the magnetosphere during SOI. This 'same' recurring compression region was associated with a strong SKR burst and auroral activity during the January 2004 HST/Cassini campaign, as described above. They have also shown a general correspondence between high field strength intervals with embedded HCS crossings and high-power SKR emissions.

[5] In Figure 1 we show the magnetic field and SKR emissions observed during SOI and the effects of the CIRrelated compression. In the top panel we show the RPWS frequency-time spectrogram of SKR emission as observed by Cassini, whilst in the bottom panel we show the total magnetic field strength during the SOI interval (days 181186). This interval encompasses the final magnetopause crossing inbound on day 181 (indicated by the vertical arrow marked 'MP' in the bottom panel) through to the 


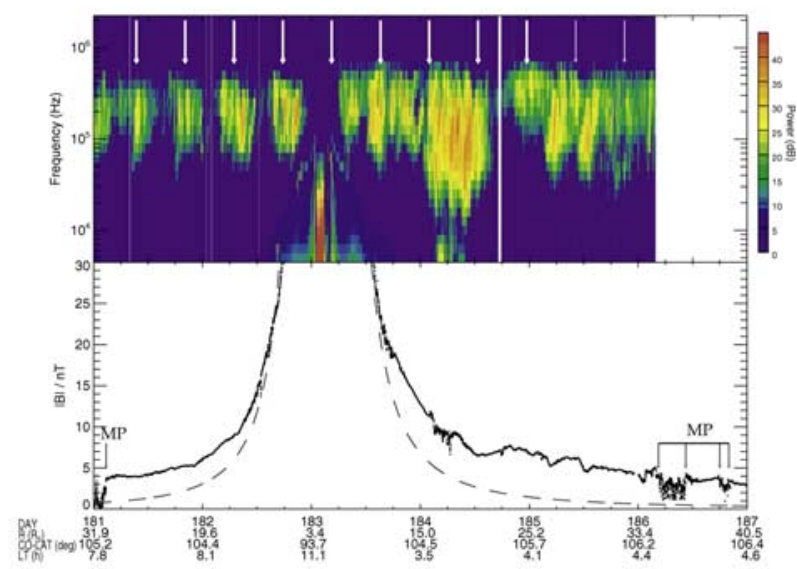

Figure 1. Overview of Cassini SKR and magnetic field observations during SOI. (top) The RPWS data shown as a frequency-time spectrogram. The white arrows are plotted every $10.75 \mathrm{~h}$, highlighting the phase of the planetary modulated emission. (bottom) We show the total magnetic field strength, with the dashed curve indicating the SPV model internal field. Vertical arrows mark magnetopause crossings. Trajectory information in the form of radial distance $\left(\mathrm{R}_{\mathrm{s}}\right)$, co-latitude $(\mathrm{deg})$, and local time $(\mathrm{h})$ is given at the bottom of the plot.

first magnetopause crossings outbound on day 186 (marked similarly), with closest approach (CA) at 02:39 UT on day 183. In the top panel, we see that planetary modulated SKR emissions spanning $\sim 40-600 \mathrm{kHz}$ are present throughout the inbound pass. A series of vertical arrows have been drawn at fixed planetary period intervals of $10.75 \mathrm{~h}$, placed to coincide with peaks in the emission inbound. An $\sim 8 \mathrm{~h}$ gap in SKR emissions then occurs near CA [Gurnett et al., 2005]. The emission resumes some $4 \mathrm{~h}$ after CA, initially in phase with that recorded on the inbound pass. A major burst of SKR emission then occurs on day 184, extending down to low frequencies and lasting for $\sim 14 \mathrm{~h}$. This burst occurs in anti-phase with the previously planetary-modulated emissions observed inbound. Following this initial burst, a period of SKR quiet was observed, before further highpower bursts ensued on day 185 (again generally out of phase with the inbound planetary modulation). These data are rather similar to those measured during January 2004, where 'out of phase' SKR bursts were associated with a high-field compression region, and major auroral activity [Kurth et al., 2005; Jackman et al., submitted manuscript, 2005].

[6] We now turn to the magnetic field strength values shown in the bottom panel of Figure 1. The dashed line indicates the internal planetary field calculated from the Saturn-Pioneer-Voyager model (SPV) of Davis and Smith [1990]. The radial distance, co-latitude of the spacecraft relative to the spin-axis, and local time are shown at the bottom of the panel. We see that Cassini entered the magnetosphere at a post-dawn local time of $\sim 7.6 \mathrm{~h}$, and exited at a pre-dawn local time of $\sim 4.5 \mathrm{~h}$. The magnetic field data from the inbound pass show no particular evidence that a sudden compression of the magnetosphere had taken place (a compression would be expected to produce an increase in the field strength of a few nT over $\sim 1 / 2$ hour). During the outbound pass, near to the middle of day 183, the spacecraft is within a region of warm middle magnetosphere plasma [Young et al., 2005], accompanied by small scale fluctuations in the field, also seen at the corresponding location inbound [Dougherty et al., 2005]. At $\sim 21: 30$ UT Cassini then entered a region of quiet field which corresponds to open field lines of the tail lobe (see the following section). Suddenly, at $\sim 02: 00$ UT on day 184 , at a radial distance of $\sim 16 \mathrm{R}_{\mathrm{s}}$, the spacecraft observed a reduction in the field strength followed by rapid fluctuations of the field. Similar, weaker events are seen to occur on day 185 . It can be seen that these fluctuations correlate directly with the SKR bursts in the top panel, and suggest tail dynamics and possible injection of hot plasma into the nightside-dawn magnetosphere, accompanied by the excitation of field-aligned currents and auroral precipitation. This we thus further investigate.

\section{In Situ Observations of Hot Plasma Injection and Field Reconfiguration}

[7] In this section we further investigate the interval between 06:00 UT on day 183 and 12:00 UT on day 186, including the plasma data from MIMI-LEMMS and CAPS-ELS. In Figure 2, the top panel indicates the approximate mapping of the spacecraft position to the northern and southern ionosphere depicted by the solid and dashed lines respectively. This mapping is estimated using the Connerney et al. [1983] model of the external field due to the ring current in Saturn's middle magnetosphere, along with the SPV internal field. The Connerney et al. [1983] model has been modified to reflect the conditions observed during the outbound portion of the SOI fly-through. In the second panel we show the RPWS time-frequency spectrogram in the same format as Figure 1, and in the third panel we show the magnitude of the magnetic field. The fourth panel shows MIMI-LEMMS observations of high-energy ions from four energy channels (A1-4) indicated by different colours. The blue line indicates ions in the range $36-53 \mathrm{keV}$, green $53-105 \mathrm{keV}$, yellow $105-235 \mathrm{keV}$, and red $493-783 \mathrm{keV}$. Finally, the fifth panel shows the CAPS-ELS measurements of electrons in the energy range $\sim 0.5-25 \mathrm{keV}$, in the form of an energy-time spectrogram. In a similar format to Figure 1, spacecraft trajectory information is shown at the bottom of the plot.

[8] On the left of Figure 2, at 06:00 UT on day 183, the spacecraft is located at a radial distance of $\sim 3.9 R_{s}$, at colatitude of $\sim 95.7^{\circ}$ in the southern hemisphere, and a local time of $\sim 1.9$ h (i.e. post-midnight). As discussed by Krimigis et al. [2005], the spacecraft was located in the radiation belts of Saturn until $\sim 12: 00$ UT on this day (marked by the vertical dashed line). After $\sim 12: 00$ UT, the spacecraft exits this inner region and enters the plasma sheet region of the middle magnetosphere $\left(>10 R_{S}\right)$. Here the magnetic field is characteristically stretched due to the presence of an azimuthal ring current, and undergoes rapid fluctuations due to the presence of warm plasma [Dougherty et al., 2005]. The LEMMS data show the presence of ions with energies of several $10 \mathrm{~s}$ of $\mathrm{keV}$, while the ELS data indicates the presence of hot electrons with energies of $\sim 1 \mathrm{keV}$. At $\sim 22: 00 \mathrm{UT}$, the magnetic field becomes 


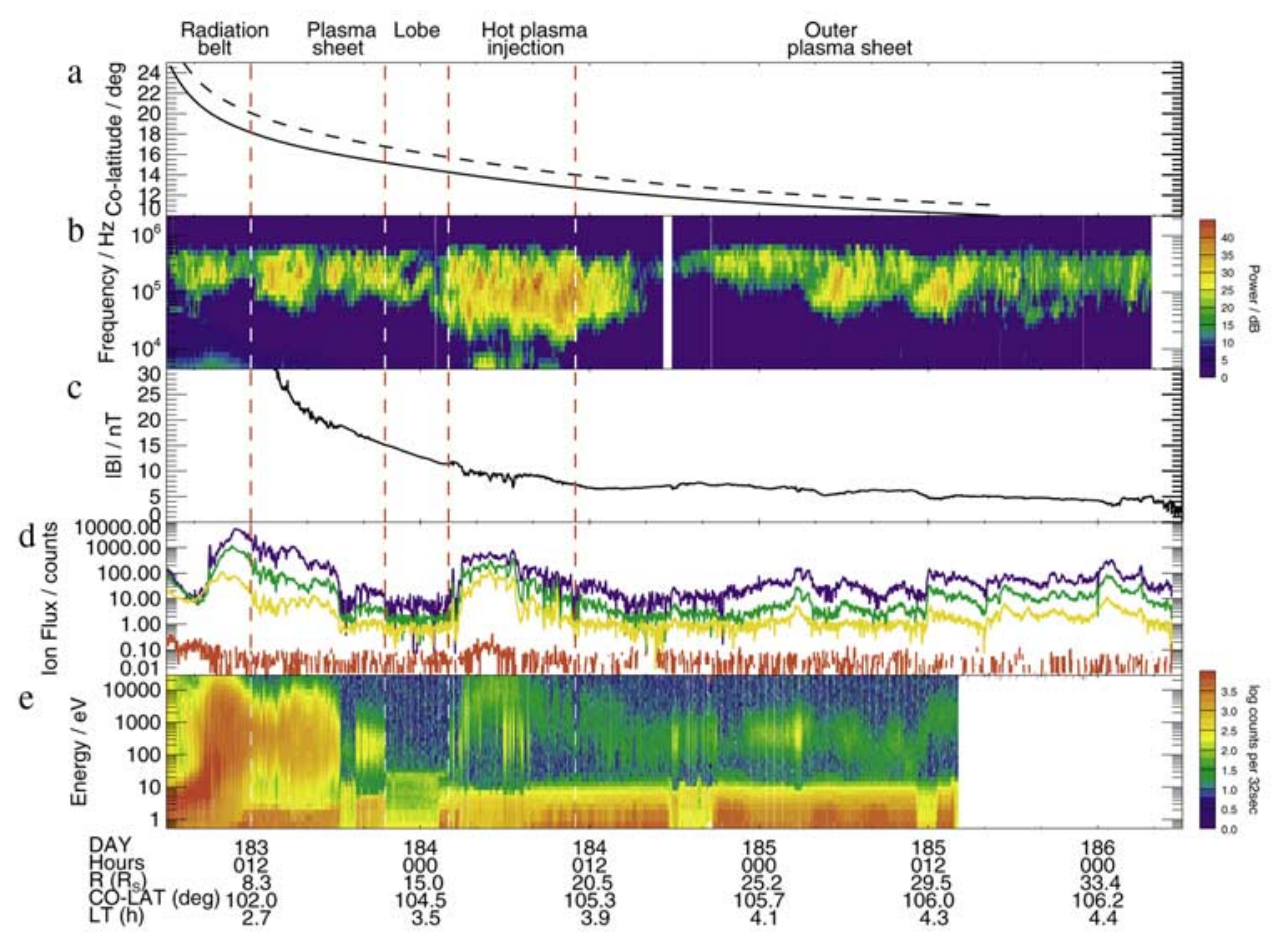

Figure 2. Stack plot of five panels from (a) the outbound SOI pass showing the co-latitude of the foot of the magnetic field line passing through the spacecraft mapped to the northern (solid line) and the southern ionosphere (dashed line) using the Connerney et al. [1983] model with $\mathrm{R}_{0}=6.5 \mathrm{R}_{\mathrm{s}}, \mathrm{R}_{1}=17.5 \mathrm{R}_{\mathrm{s}}, \mathrm{D}=1.75 \mathrm{R}_{\mathrm{S}}$ and $\mu_{0} \mathrm{I}_{0}=90.2 \mathrm{nT}$, (b) the RPWS frequency-time spectrogram, (c) the magnetic field strength data, (d) four energy channels of MIMI-LEMMS ions, and (e) a CAPS-ELS energy-time spectrogram. The vertical dashed lines indicate the boundaries of magnetospheric regions.

abruptly quiet and the hot ion and electron populations essentially disappear. This signature is indicative of open field lines in the tail lobe. The mapping of the field at this time (panel 1) gives a co-latitude of $\sim 14.5^{\circ}$ in the northern ionosphere, and $\sim 16^{\circ}$ in the southern ionosphere. These values are typical of a location just poleward of the open-closed field line boundary, judging from HST auroral images [e.g., Grodent et al., 2005].

[9] At $\sim 02: 00$ UT on day 184 when the spacecraft is at a radial distance of $\sim 16 \mathrm{R}_{\mathrm{s}}$ and a local time of $\sim 3.6 \mathrm{~h}$, the spacecraft becomes engulfed in hot plasma (panels 4 and 5), accompanied by a reduction in field strength and field fluctuations (panel 3). This occurs simultaneously with the SKR burst seen in panel 2. The LEMMS ion data show an abrupt increase in flux in all energy channels with a spectrum which is considerably harder than in the previous plasma sheet intervals. Concurrently, the ELS electron spectrogram shows the arrival of a hot, tenuous electron population with energies up to at least $25 \mathrm{keV}$. After a few hours the plasma cools and the fluxes subside, but the spacecraft appears to remain within this tail plasma sheet population, possibly its outer layer, for the entirety of the outbound pass, as identified by Krimigis et al. [2005]. One explanation of these events is that the enhanced particle fluxes observed on day 184 are consistent with an injection of heated plasma from a downtail reconnection site, as suggested by Cowley et al. [2005]. Two further enhancements are seen in the SKR power during the first half of day 185 , accompanied by depressions in the field magnitude. These secondary effects appear to relate to fluctuations in the energy and density of both the LEMMS ions and ELS electrons, perhaps indicating further 'bursts' of reconnection in the tail.

[10] Finally, we examine the magnetic field response during the hot plasma injection identified above. In

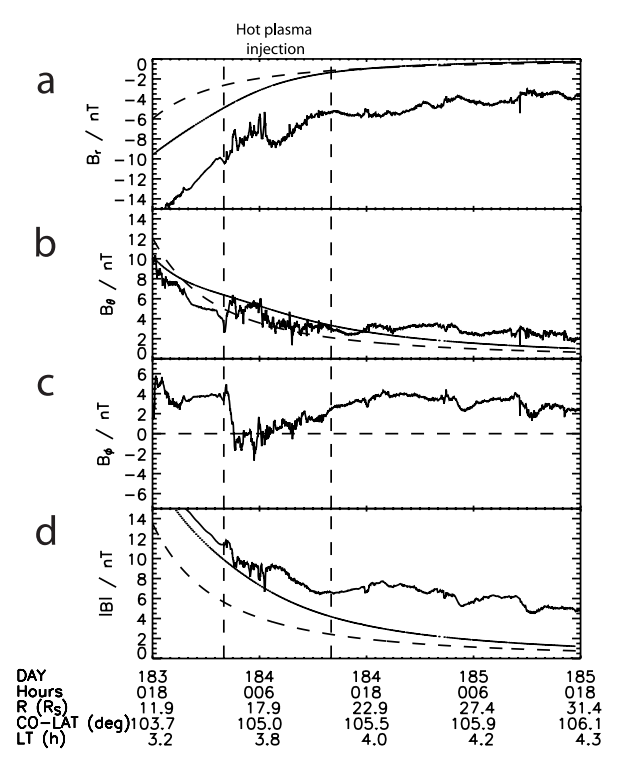

Figure 3. Plot of the magnetic field components in spherical polar coordinates referenced to Saturn's spin (and magnetic) axis: (a) $B_{r}$, (b) $B_{\theta}$, and (c) $B_{\varphi}$ components. (d) The total magnetic field strength. The dashed line shows the SPV model values, whilst the solid line shows the modified Connerney model as in Figure 2. 
Figure 3 we show in the top three panels the magnetic field components in a spherical polar coordinate system relative to Saturn's spin axis, with the total field strength shown in the fourth panel. In a similar format to Figure 1, the internal planetary field of the SPV model is shown by the dashed line, while the solid line depicts the modified Connerney model discussed in relation to Figure 2. As in previous figures, the location of the spacecraft is shown at the bottom of the plot. Here we can clearly see the 'hot plasma injection event' on day 184, beginning at $\sim 02: 00 \mathrm{UT}$ and lasting for $\sim 9 \mathrm{~h}$. Initially, over the first $30-60 \mathrm{~min}$, the total field strength increases by $\sim 1 \mathrm{nT}$. This signature may signify the effect of the shockcompression impacting the magnetosphere. Following this the total field strength is reduced by $\sim 3 \mathrm{nT}$, corresponding to a decrease in the magnetic pressure by $\sim 40 \%$, accompanied by considerable fluctuations indicative of the presence of hot plasma. During this reduction in field strength, the azimuthal component of the field turns from positive values (a tailward pointing orientation), to near-zero values (a relaxed meridional field in the planetary direction). In addition, the magnitude of the radial field decreases whilst the latitudinal component increases. These signatures are consistent with a relaxation of the field from a tail-like to a more planetary-like orientation, similar to 'dipolarisations' observed at Earth. We suggest that the injection of hot plasma and concurrent field relaxation, occurring in tandem with a major SKR burst, is likely related to magnetic reconnection and closure of flux in Saturn's magnetotail. Overall, we suggest these data show the first in situ observations of compression-related dynamics in Saturn's magnetotail.

\section{Summary}

[11] A major compression of Saturn's magnetosphere took place during the Cassini SOI fly-through of Saturn's magnetosphere (Jackman et al., submitted manuscript, 2005). For the first time, we have witnessed the in situ effects that the CIR-related compression has on Saturn's magnetospheric dynamics. At $\sim 02: 00$ UT on day 184, a burst of SKR emission is observed which disrupts the existing pattern of planetary modulated emission seen both upstream of the magnetosphere and during the inbound pass [Kurth et al., 2005; Jackman et al., submitted manuscript, 2005]. Simultaneously, inside the magnetosphere, Cassini experienced a region of depressed and variable magnetospheric field. In addition, ion and electron observations show that this occurs as the spacecraft is engulfed by a hot, tenuous plasma population. While subsequently cooling, the spacecraft remained within this plasma sheet population for the duration of the outbound pass. We have thus shown that following the shock-compression, the magnetosphere underwent a significant reconfiguration, exemplified by a relaxation of the field and an injection of hot plasma. We propose that this behaviour is indicative of a major episode of tail reconnection, triggered by the impact of the compression region on Saturn's magnetosphere, as discussed in relation to the January 2004 HST-Cassini interval by Cowley et al. [2005].

\section{References}

Boudouridis, A., E. Zesta, R. Lyons, P. C. Anderson, and D. Lummerzheim (2003), Effect of solar wind pressure pulses on the size and strength of the auroral oval, J. Geophys. Res., 108(A4), 8012, doi:10.1029/ 2002JA009373.

Bunce, E. J., et al. (2005), Cassini magnetometer observations of the solar wind upstream of Saturn and their relation to Hubble Space Telescope aurora data, Adv. Space. Res., in press.

Clarke, J. T., et al. (2005), Morphological differences between Saturn's ultraviolet aurorae and those of Earth and Jupiter, Nature, 433, 717.

Connerney, J. E. P., M. H. Acuña, and N. F. Ness (1983), Currents in Saturn's magnetosphere, J. Geophys. Res., 88, 8779.

Cowley, S. W. H., S. V. Badman, E. J. Bunce, J. T. Clarke, J.-C. Gérard, D. Grodent, C. M. Jackman, S. E. Milan, and T. K. Yeoman (2005), Reconnection in a rotation-dominated magnetosphere and its relation to Saturn's auroral dynamics, J. Geophys. Res., 110, A02201, doi:10.1029/ 2004JA010796.

Crary, F. J., et al. (2005), Solar wind dynamic pressure and electric field as the main factors controlling Saturn's aurorae, Nature, 433, 720.

Davis, L., Jr., and E. J. Smith (1990), A model of Saturn's magnetic field based on all available data, J. Geophys. Res., 95, 15,257.

Dougherty, M. K., et al. (2005), Cassini magnetometer observations during Saturn orbit insertion, Science, 307, 1266.

Grodent, D., J.-C. Gérard, S. W. H. Cowley, et al. (2005), The global morphology of Saturn's southern ultraviolet aurora, J. Geophys. Res., doi:10.1029/2004JA010983, in press.

Gurnett, D. A., et al. (2005), Radio and plasma wave observations at Saturn: Initial results from Cassini, Science, 307, 1255.

Jackman, C. M., N. Achilleos, E. J. Bunce, S. W. H. Cowley, M. K. Dougherty, G. H. Jones, S. E. Milan, and E. J. Smith (2004), Interplanetary magnetic field at $<9$ AU during the declining phase of the solar cycle and its implications for Saturn's magnetospheric dynamics, J. Geophys. Res., 109, A11203, doi:10.1029/2004JA010614.

Krimigis, S. M., et al. (2005), The dynamic Saturn magnetosphere: First results from Cassini/MIMI, Science, 307, 1270.

Kurth, W. S., et al. (2005), An Earth-like correspondence between Saturn's auroral features and radio emission, Nature, 433, 722.

Young, D., et al. (2005), Composition and dynamics of plasma in Saturn's magnetosphere, Science, 307, 1262.

E. J. Bunce, S. W. H. Cowley, and D. M. Wright, Department of Physics and Astronomy, University of Leicester, Leicester LE1 7RH, UK. (emma.bunce@ion.le.ac.uk)

A. J. Coates and A. M. Rymer, Mullard Space Science Laboratory, University College London, Dorking RH5 6NT, UK.

M. K. Dougherty, Blackett Laboratory, Imperial College, London SW7 2BZ, UK

N. Krupp, Max-Planck-Institut für Sonnensystemforschung, D-37191 Katlenburg-Lindau, Germany.

W. S. Kurth, Department of Physics and Astronomy, University of Iowa, Iowa City, IA 52242, USA. 